\title{
EVALUATION OF THE RESULTS OF DIFFERENT PROCEDURES IN COCHLEAR IMPLANTATION.
}

\author{
By
}

Alaa El-Din M El-Feky ${ }^{a}$ Ezz El-Din M El Shiekh ${ }^{a}$; Hassan Wahba ${ }^{b}$

and Ahmed s El Aassar ${ }^{a *}$

a: Otorhinolaryngology Department, Faculty of Medicine, Zagazig University

b: Otorhinolaryngology Department, Faculty of Medicine, Ain-Shams University

\begin{abstract}
Background: The cochlear implantation has radically changed the outlook for profoundly deaf adults and children. The cochlear implant can provide sufficient hearing sensations to enable most severely or profoundly deafened persons to continue communicating using speech as their primary means of communication .In Classic cochlear implantation; mastoidectomy is done to help retain the electrode leads within the confines of the mastoid cavity. Then the facial recess should be widely opened followed by cochleostomy which is followed by insertion of the electrode array. Minimally invasive cochlear implant surgery has become the mainstay of most experienced centers. Aim of work: to evaluate the results of using different procedures in cochlear implantation. Patients and methods: The study carried out on 41 patients underwent cochlear implantation, starting from April 2011 till April 2014. Patients were 27 males and 14 females, their age ranged from 2 years to 18 years and all complained of profound sensori-neural hearing loss.

We excluded postlingual adults $(n=2)$ and children with congenital anomalies $(n=3)$, post-meningitis cochlear ossification $(n=1)$ and children with chronic suppurative otitis media $(n=1)$. Total number of excluded cases is 7 patients and remains 34 patients. The patients were divided into two groups: the 1st group of patients were implanted by classic approach (n=28) the 2nd group of patients were implanted by the SMA $(n=6)$ and another comparison in which, the patients were divided into patients was implanted by PULSAR ci100 device (5 cases) and the patients were implanted by HI-RES $90 \mathrm{~K}$ device (29cases); all patients were subjected to full preoperative assessment, and full postoperative assessment of complications (major and minor complications). Results: there is significant relation between the two groups regarding major complications in favor to classic approach but there is significant danger of facial nerve, chorda tympani nerve injury in classic approach, there is no significant difference between the ways of insertion regarding the major complications and there is significant difference between the 2 groups as regard to device migration, electrode extrusion and device malfunction in favor to Hi-res 90k device. Conclusion: classic approach has fewer incidences of major and minor complications than SMA, but there is significant danger of facial nerve, chorda tympani nerve injury in classic approach, Hi-res 90k device is better than PULSAR ci100 device as regard to device migration, electrode extrusion and device malfunction, We still need more assessment for surgical approaches, way of electrode insertion, type of devices.

Keywords: Cochlear implantation, Suprameatal approach, Posterior tympanotomy approach.
\end{abstract}

\section{INTRODUCTION}

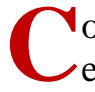
ochlear implant is a device that delivers electrical stimulation through an array of electrodes to a bundle of cochlear nerve fibers. It is established as an effective and safe method of rehabilitation for profoundly deaf patients 1

Cochlear implants are the first true bionic sense organs. The human cochlea is an electromechanical transducer. Cochlear implants, like other human hair cell, receive mechanical sound energy and convert it into a series of electrical impulses $\mathbf{2}$

Sound is first detected by a microphone (usually worn on the ear) and converted into an analog electrical signal. This signal is then sent to an external processor where it is transformed into an electronic code. This code is transmitted via radiofrequency across the skin by a transmitting coil. Ultimately, this code is translated by the receiver-stimulator into rapid electric impulses distributed to electrodes on a coil implanted within the cochlea 3

Several cochlear implants are commercially available in the market and are manufactured by Cochlear Corporation, Advanced Bionics, and the Med El Corporation. Over the years, subsequent generations of the various components of the devices have been approved by the U.S. Food and Drug Administration (FDA), focusing on improved electrode design and speech-processing capabilities. Furthermore, smaller devices and the accumulating experience in children have resulted in broadening of the selection criteria to include children as young as 6 months 4

The candidacy for implantation is considered separately for adults and children. As outlined in the 1995 National Institutes of Health (NIH) consensus statement on cochlear implantation, adult candidacy is noted as being successful in postlingually deaf adults with severe-to-profound hearing loss with no speech perception benefit from hearing aids. 
Prelingually deafened adults must be counseled in regard to realistic expectations, as language and open-set speech discrimination outcomes are less predictable. Children are considered candidates for implantation at age 6 months because of meningitisrelated deafness with progressive cochlear ossification. Also, audiological criteria include severe-to-profound sensorineural hearing loss bilaterally and poor speech perception under bestaided conditions, with a failure to progress with hearing aids and an educational environment that stresses oral communication $\mathbf{5}$

The classic surgery involves mastoidectomy, posterior tympanotomy, cochleostomy, and insertion of array of electrodes through the basal coil of the cochlea. General anesthesia is needed for children and is usual for adult. The body of the implant is inserted into a seat drilled in the skull behind the ear 6

Minimal access surgery for cochlear implantation has been developed in recent years in order to decrease surgical trauma and secondary complications, mostly related to size and shape of the skin flap.

Although cochlear implantation is considered a safe method of habilitation / rehabilitation for profoundly deaf individuals, a number of these patients suffer complications after surgery $\mathbf{7}$.

Surgical complications may be classified into major (if they require additional surgery or hospitalization), and minor, (when they resolve with treatment in an outpatient ward or even with no treatment at all). Major complications involve meningitis, flap necrosis, device failure, electrode extrusion, facial nerve paralysis and others; while the minor complications involve facial nerve stimulation, electrode migration, vertigo, tinnitus, and others. The major surgical complications which require surgery review and, especially those associated with device insertion are not common $\mathbf{8}$

\section{Patients and methods}

Selection of patients

From April 2011 till April 2014, 41 patients underwent cochlear implantation surgery in Zagazig University were included in the study. Patients were 27 males and 14 females, their age ranged from 2 years to 18 years $($ mean $=5 \pm$ S.D $),(S . D=1.81)$ and all complained of profound sensori-neural hearing loss.

Selection criteria:
- Patients suffer from bilateral SNHL with no measurable benefit from hearing aids over a time period of 6 months.

- Realistic expectations by the patients and their parents.

- No psychological contra indication for surgery. Exclusion criteria:

- Adults suffer from moderate to severe sinsori-neural hearing loss.

- Unilateral hearing loss.

- Patients underwent previous mastoid surgery at the same ear.

- Patients suffer from congenital anomalies in the inner ear.

- Patients suffer from post meningitis cochlear ossification.

We excluded postlingual adults $(n=2)$ and children with congenital anomalies $(n=3)$, postmeningitis cochlear ossification $(n=1)$ and children with chronic suppurative otitis media $(n=1)$. Total number of excluded cases is 7 patients and remains 34 patients.

They all underwent:

1- Pre-operative evaluation

All patients in the study had done basic preoperative assessment, which include the following:

- Thorough general examination and ENT examination

- Written consents were taken from all cases

- Audiological assessment

- Tympanometry.

- Aided and non-aided audiometry by either play audiometry or visual reinforcement audiometry.

- Auditory brainstem response

- OtoAcoustic Emission

- Language assessment

- Intelligence Quotient (IQ) assessment

- Electroencephalogram (EEG)

- Imaging

o Computerized tomography (CT scan) of the temporal bone

- Magnetic resonance imaging (MRI) of the cochlea, auditory nerve and brain

2- Surgical techniques

A. The classic approach

1. Incision: 2 types of incisions:

- Post auricular inverted J shaped incision: in7cases

- Extended endaural incision: in 21 case 


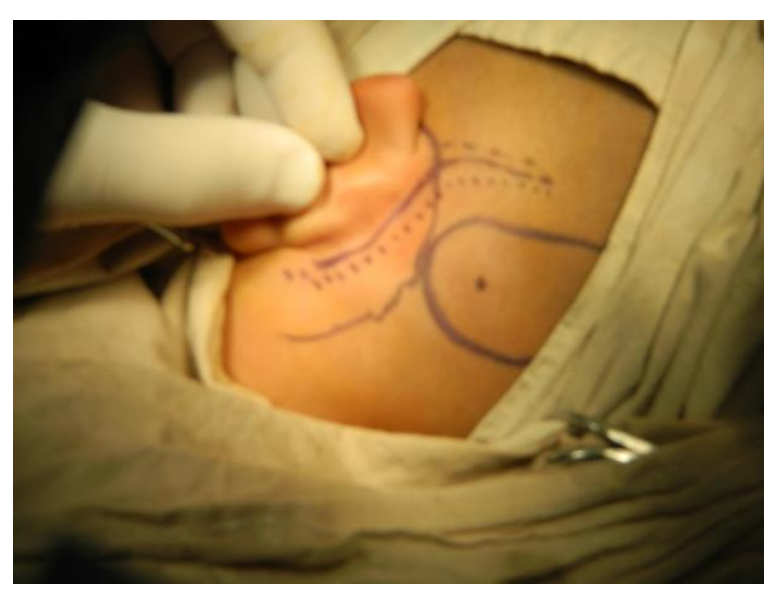

Fig (1) post auricular inverted $\mathbf{J}$ shaped incision

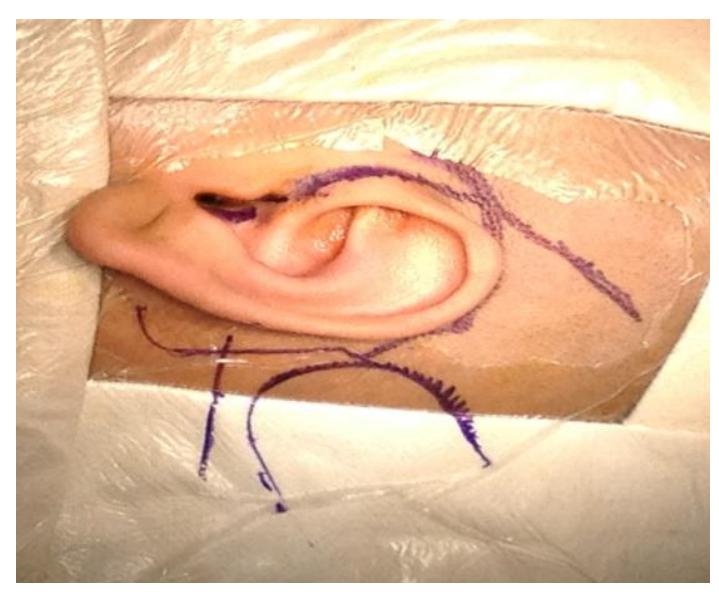

Fig (2): extended endaural incision

\section{Elevation of the flap 2 layer}

2 flaps layers are elevated

1. The superficial layers include skin and S.C tissue.

2. The deep layer:
- Anterior based Palva flap

- Upper flap is elevated in a sub-periosteal plane to create device seat.

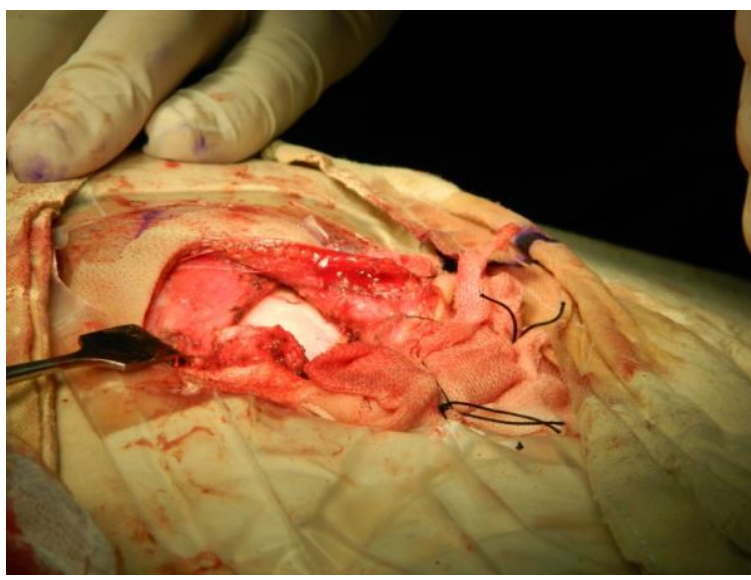

Fig (3) Palva flap

\section{Mastoidectomy and Posterior tympanotomy}

The facial recess opening is lowered down and the bone anterior to $\mathrm{FN}$ is removed till good exposure to round wind niche is achieved.

\section{Cochleostomy or RW approach}

- First we used to do cochleostomy by opening cochlea in the promontory antro-inferior to round window niche with $1 \mathrm{~mm}$ diamond bur.

- Now we used to open the RW membrane in all cases, except if difficult exposure, by good removing of entire RW niche till good exposure of RW membrane.

\section{Creation of the receiver/stimulator seat}

6. Electrode insertion

7. Muscle Plug

8. Fixation

$\circ$ We depend in fixation on sewing the periosteum together over the implant.

9. Intraoperative $\mathrm{X}$-ray (C-arm) 


\section{(1) 0ณ1}

Fig (4) Intraoperative X-ray

10. Suturing

11. Intraoperative device function assessment

12. Dressing B- Suprameatal technique

1- Incision: extended endaural

2- Elevation of the outer flaps

3- Creation of the seat

4- Elevation of tympanomeatal flap and anterior tympanotomy.

5- Creation of the groove
- A groove is made in in postro superior wall of EAC starting from inside to outside. The groove is $1 \mathrm{~mm}$ width and $3 \mathrm{~mm}$ in depth. It started from the scutum lateral to the long process of incus and just above the level of the pyramid, the chorda tympani should be identified before making the groove and reflected anteriorly away from the groove.

- The groove is continued in outer direction till it reaches the site of the seat without doing the blind tunnel as in classic SMA.

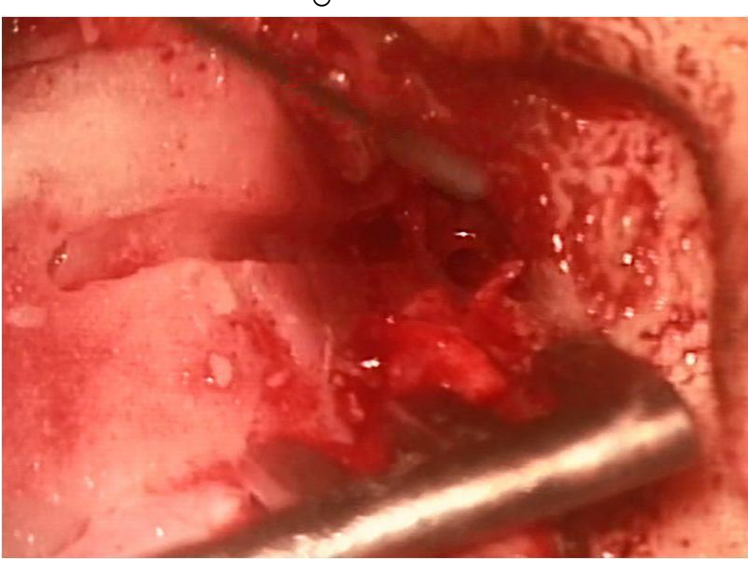

Fig (5) creation of the groove

\section{6- Exposure of RW}

- In all these cases the RW membrane was good exposed after removing of entire RW niche.

\section{7- Electrode insertion}




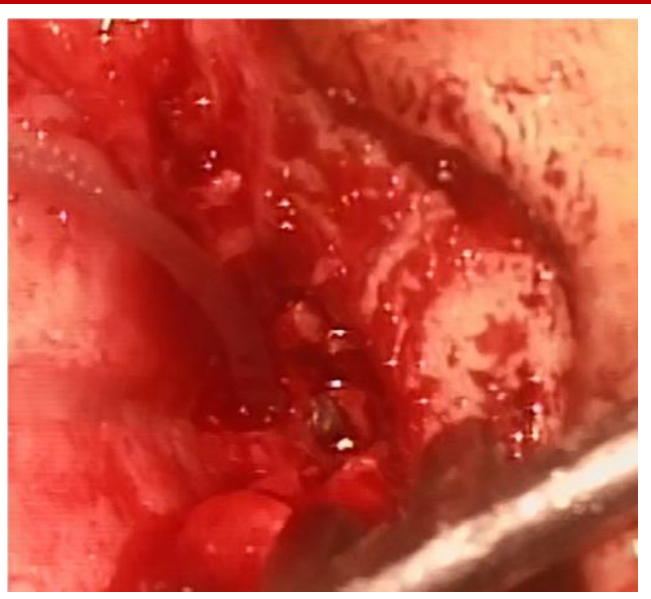

Fig (6): Electrode insertion

8- Fixation of electrode and obliteration of the groove.

By either
- Cartilage and cement like material (glass ionomer or calcium hydroxide).

- bone Pate and bioactive glass powder

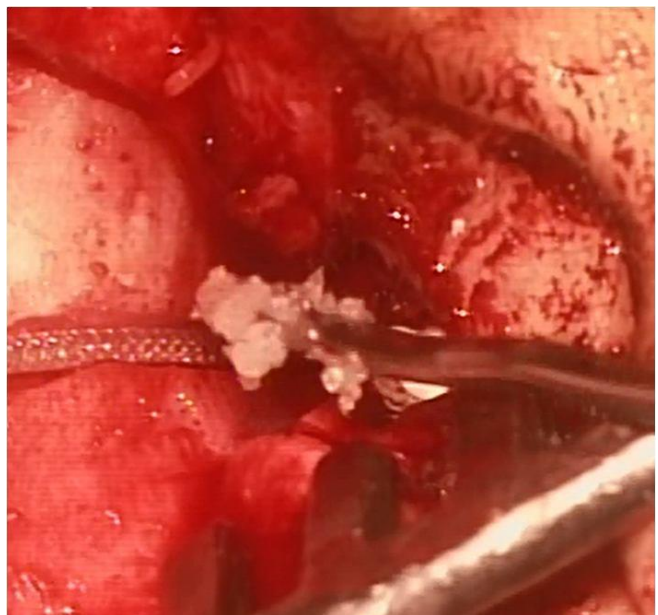

Fig (7): Electrode fixation using bioactive glass

\section{9- Grafting and Packing with gelfoam}

- Securing of the TM by temoralis fascia graft, even if intact TM, which is supported by gelfoam in M.E

- Then Tympanomeatal flap is returned and supported by gelfoam and one aural pack to be removed after 2 weeks.

10- Intraoperative X- ray (C- arm)

11- Intraoperative device function assessment

12- Dressing

3- Postoperative care:

- 1 st 24 hour we concern on the following: Facial nerve function, Vomiting, Vertigo, Temperature and Dressing is soaked or not

- $\quad$ After 24 hour, we evaluate the presence or absence of hematoma, if hematoma is present, it should be evacuated by aspiration under complete aseptic condition. Then we do digital x ray, Stenver view, for documentation, then we discharge the patient. on oral antibiotic
- After 1 week, we stop the antibiotics and remove the dressings, steristrips and cutaneous sutures, if present.

- After 4 weeks, we start external device programing and recording any twitches, significant pain, and then we start Speech rehabilitation sessions.

- $\quad$ After 2 month, Routine follow-up is done every 2 months

4- Statistical Analysis:

Statistical analyses were performed using SPSS 14.0 statistical software for Windows (SPSS Inc, Chicago, IL). The significance level was set at P\0.05 with a confidence a level $95 \%$. The data of the patients, prostheses and procedures were collected, arranged and tabulated then compared using a t test for quantitative data and chi square test for qualitative data. 
RESULTS

Table (1): Age groups distribution

Age and sex distribution

\begin{tabular}{|c|c|c|}
\hline Age group & No. & $\%$ \\
\hline $2-<4$ years & 8 & 24 \\
\hline $4-<6$ years & 24 & 70 \\
\hline 6-7 years & 2 & 6 \\
\hline Total & 34 & 100 \\
\hline \multicolumn{3}{|c|}{ Table (2): sex distribution } \\
\hline Sex & No. & $\%$ \\
\hline Male & 22 & 65 \\
\hline Female & 12 & 35 \\
\hline Total & 34 & 100 \\
\hline
\end{tabular}

Table (3): shows group with one or more minor surgical complication:
Minor complications
$\mathrm{A}(28$ cases $)$
$\mathrm{B}(6$ cases $)$

\begin{tabular}{|c|c|c|c|c|}
\hline \multirow{2}{*}{ 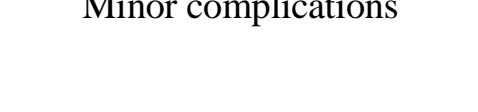 } & \multirow[b]{2}{*}{ Total } & \multirow[b]{2}{*}{ Percentage } & \multirow[b]{2}{*}{ Total } & \multirow[b]{2}{*}{ Percentage } \\
\hline & & & & \\
\hline $\begin{array}{ll}\text { Chorda tympani nerve } \\
\text { Injury }\end{array}$ & 6 & $21.4 \%$ & 1 & $16.6 \%$ \\
\hline Fever & 4 & $14.3 \%$ & 1 & $16.6 \%$ \\
\hline Vomiting & 5 & $17.9 \%$ & 1 & $16.6 \%$ \\
\hline Vertigo & 3 & $10.7 \%$ & 1 & $16.6 \%$ \\
\hline Hematoma & 4 & $14.3 \%$ & 0 & $0 \%$ \\
\hline Wound infection & 2 & $7.1 \%$ & 1 & $16.6 \%$ \\
\hline Device migration & 3 & $10.7 \%$ & 1 & $16.6 \%$ \\
\hline EAC stenosis & 0 & $0 \%$ & 1 & $16.6 \%$ \\
\hline $\begin{array}{l}\text { Total cases with one or } \\
\text { more minor complications }\end{array}$ & 11 & $39.3 \%$ & 3 & $50 \%$ \\
\hline $\begin{array}{l}\text { hi squire }=3.8141 \\
=0.0431 \text { (significant })\end{array}$ & & & & \\
\hline
\end{tabular}

As shown in Table (3), there is significant relation in favor to group (A) regarding postoperative minor complications, but there is more danger of chorda tympani nerve injury in group (A) (patients underwent cochlear implantation by classic approach). 
Table (4): shows group with one or more major surgical complication:

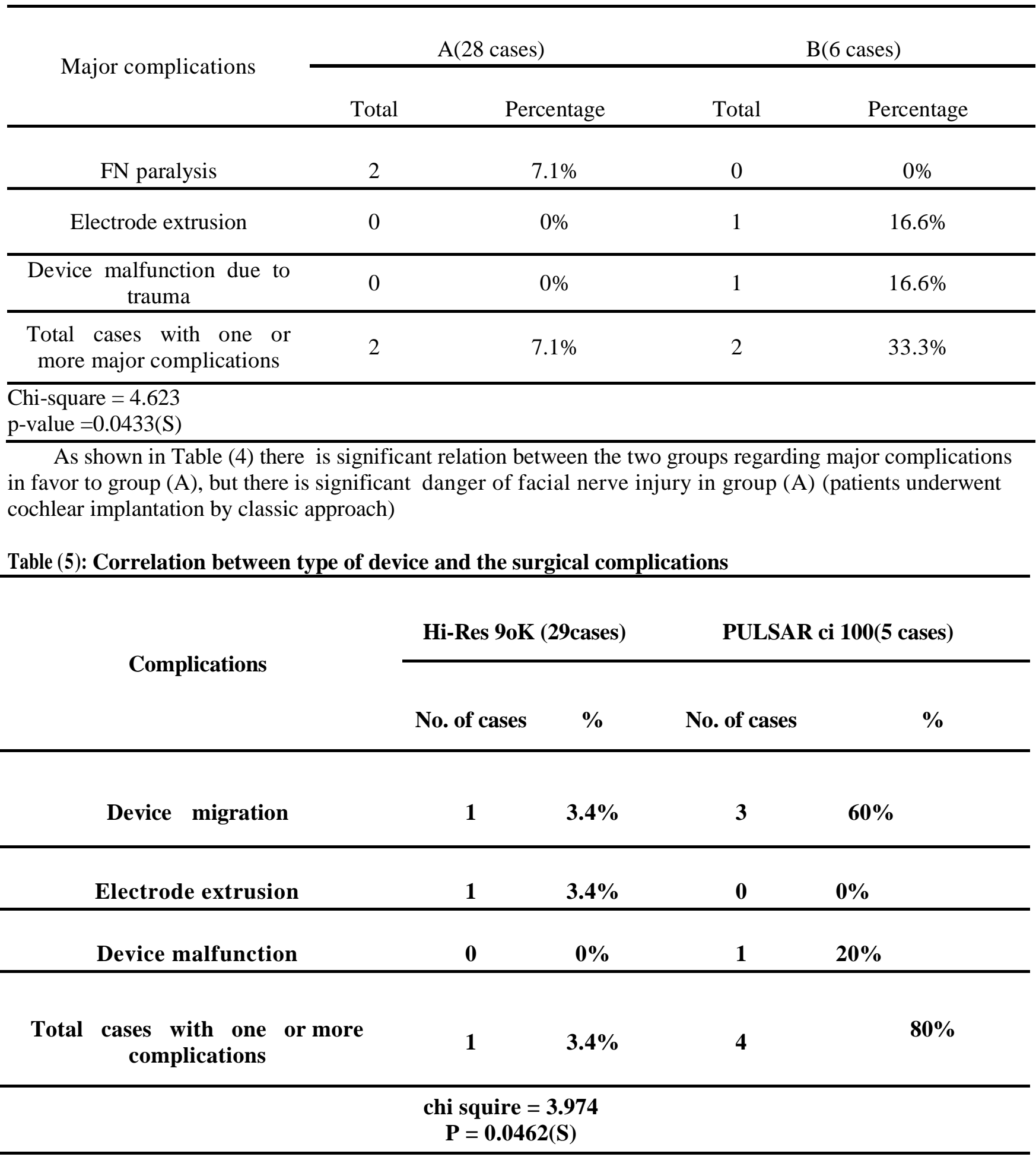

As shown in table (5) there is significant difference between the 2 groups as regard to device migration, electrode extrusion and device malfunction in favor to group B.( Hi-res 90k advantage). 


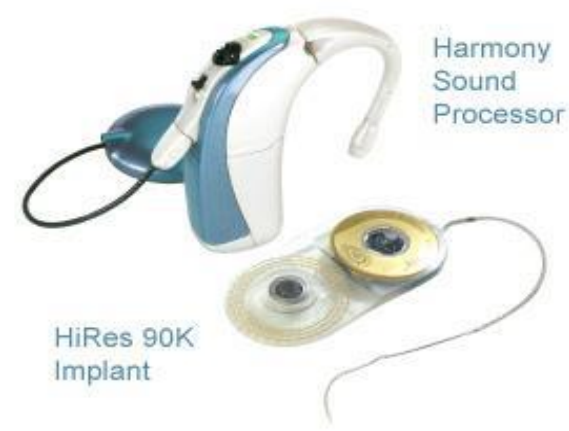

Fig (8): The Harmony ${ }^{\mathrm{TM}}$ sound processor and HiRes $90 K^{\mathrm{TM}}$ implant from Advanced Bionics

\section{DISCUSSION}

This study revealed 6 cases (21.4\%) suffered from chorda tympani injury \{ seen during operation without any postoperative manifestations \} out of 28 cases underwent cochlear implantation by classic approach and one case (16.6\%) suffered from chorda tympani injury out of 6 cases underwent cochlear implantation by SMA. This is matched with Hoffman RA and Cohen NL.

Damage to the chorda tympani nerve in the classic approach was described in $5.2 \%$ to $20 \%$ of cases. While other surgeons who have reported no chorda tympani nerve injury by SMA or its modifications 7,9,10,11,12.

Three cases $(10.7 \%)$ had device migration \{ One of them cause no any symptoms, only the device is lower than its normal position, In the second case, the device cause protrusion of the auricle forward, with significant cosmetic deformity, also the patient complain from difficult hanging the external microphone on the auricle, The last case the migrated device was very low and it was not attached to the magnet of the external device well, and by using a stronger magnet, skin reaction start to appear, so the patient was revised under G.A and repositioning of the device in the seat and fixation of the device was done.\}, one case $(16.6 \%)$ suffered from device migration \{ the device migrate antro-inferiorly and become below the incision, so the patient was revised under G.A and repositioning of the device in the seat and fixation of the device was done., leading to electrode extrusion \}.This is not matching with other studies which reported no cases of electrode extrusion or damage in the following literatures'. The electrode extrusion or migration of electrode array may occur a long time after surgery. The

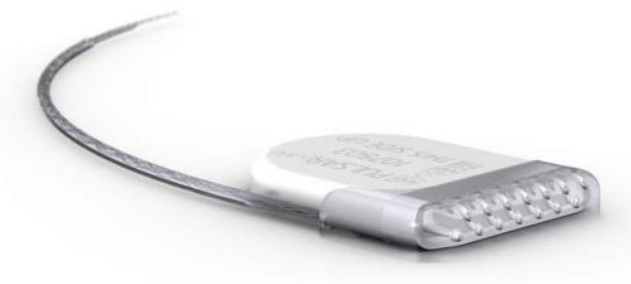

Fig (9): The PULSAR ci100 MED-El device

cause of this complication is variable, but the split bridge technique, tight packing around the cochleostomy window, or canal wall reconstruction should decrease the occurrence of this complication 11, 12, 14, and 15. This is matching with Kevin D. Brown MD, Sarah S who reported that the incidence of electrode migration is $(9 \%)$ and receiver/stimulator migration is (7\%) out of 806 cases. 13.

Two cases $(7.1 \%)$ suffered from facial nerve paralysis \{one patient didn't improve; this may be also due to difficult steroid therapy as the patient was diabetic with unstable blood glucose level. Physiotherapy was started to avoid muscle atrophy (Died 25days post-operative from hypoglycemic coma), the other patient showed mild facial nerve palsy improved after 3 weeks by steroid therapy $\}$ out of 28 cases underwent cochlear implantation by classical approach, no cases has facial nerve injury out of 6 cases underwent cochlear implantation by SMA. This is matched with other surgeons who reported no cases of FN paralysis during the SMA or its modifications7, 9,10,11,12.

One case $(16.6 \%)$ suffered from device malfunction \{due to trauma\} out of 6 cases underwent cochlear implantation by SMA, no cases has device malfunction out of 28 cases underwent cochlear implantation by classic approach..This is not matching with Kevin D. Brown MD, Sarah S who reported that the most common reasons for revision were device failure $(78 \% ; 55 \%$ hard failure, $23 \%$ soft failure) out of 805 cases $\mathbf{1 3}$.

In this study, only two types of devices had been used (Med-El PULSARci100 ffor 5 cases out of 34 cases $\}$ and Advanced bionics Hi-Res 90Kadvantage) \{for 29 cases out of 34 cases 
3devices out of 5 PULSARci100 (60\%) showed device migration, and one (20\%) showed malfunction due to trauma, on the other hand, only one device out of 29 Advanced Bionics Hi-Res 90K (3.4\%) showed device migration and when repositioned electrode has been extruded, total device failure was in one case out of $34(3 \%)$.

We found significant difference favoring Advanced Bionics Hi-Res $90 \mathrm{~K}$ regarding device migration and malfunction.

Fayad, Haensel and their colleagues reported $0.8-15.8 \%$ failure rate depending on the type of devices, and Parisier and coworkers reported an overall $11 \%$ device failure rate based on survey rate of 1175 adult patients who received multichannel implants. A higher device failure rate in children has been reported by Fayad and coworkers. Jeyakumar and Clary reported $3.0 \%$ device failure rate in pediatric cochlear implant after one year follow-up16.

Some of the speech coding in the Advanced Bionics one is a little better than the Cochlear brand. We also found out that in kids the MED-EL PULSAR ci 100device tends to breaks more often 17

\section{CONCLUSIONS}

Patients underwent cochlear implantation via posterior tympanotomy approach showed higher incidence of facial nerve and chorda tympani nerve injuries and fewer incidences of minor and major complications than patients underwent cochlear implantation via suprameatal approach.

Patients underwent cochlear implantation using $\mathrm{AB}$ Hi-Res90K advantage device have less incidence of device migration, electrode extrusion and device malfunction than others using Med-El PULSARci100 device.

We still need more assessment for surgical approaches, way of electrode insertion, type of devices and special cases that suffer from congenital anomalies, otitis media, and labyrinthitis ossificans.

\section{REFERENCES}

1. Kim CS, Chang SO, Oh SH, and Lee HJ. Complications in cochlear implantation. International congress series2004; 1273: 145-148.

2. Roland PS. Cochlear Implants, In: Glasscock ME, and Gulya AJ, (eds) SURGERY OF THE EAR, Fifth edition, Ontario, Bc Decker Inc.2003, 576-612.

3. Driscoll CL, Gluth MB, and Lalwani AK. Cochlear implants, In: Lalwani AK (ed) Current Diagnosis \& Treatment otolaryngology-Head \& Neck surgery, volume 2, New York, The McGraw-Hill companies.2004; 937947.
4. Özdemir S, Tuncer Ü, Tarkan Ö, Kıroğlu M, Çetik F, Akar F. Factors contributing to limited or non-use in the cochlear implant systems in children: 11 years experience. Int J Pediatr Otorhinolaryngol. 2013; 77(3):407-409.

5. Megerian CA and Murry GS. Cochlear Implant Surgery Workup. medscape 2013.

6. Gibson WPR. Cochlear Implants, In: Kerr AG, and Booth JB (eds). Scott-Brown's Otolaryngology, sixth edition, Oxford, Butterworth-Heinemann.1997; 3/25/13/25/20.

7. Postelman JT, Cleffken B, and Stokroos RJ. Postoperative complications of cochlear implantation in adults and children: five years' experience in Maastricht. The Journal of Laryngology \& Otology 2007; 121:318323.

8. Júnior L, Júnior F, Calhau C, Calhau A, and Palhano C. postoperative Complications in implanted patients in the Cochlear Implant Program of Rio Grande do Norte Brazil, Braz J Otorhinolaryngol.2010;76(4):517-21.

9. Hoffman RA and Cohen NL. Complications of cochlear implant surgery. Ann Otol Rhinol Laryngol Suppl. 1995; 166:420-422.

10. Hausler R: Cochlear implantation without mastoidectomy: The pericanal electrode insertion technique. Acta Otolaryngol 2002; 122: 715-719.

11. Kronenberg J. The suprameatal approach: An alternative surgical approach to cochlear implantation. Otology and Neurotology 2004; 25(1): 41-45.

12. Shankai Yin, Zhengnong Chen, Yaqin Wu, Line Wang, Jian Zhang, Wensheng Zhou, Weidong Zhou, Jiayun Huang, Zhisen Shen, Jianxin Qiu. Suprameatal approach for cochlear implantation in 45 Chinese children. International Journal of Pediatric Otorhinolaryngology 2008; 72: 397-403.

13. Kevin D. Brown MD, Sarah S. Incidence and indications for revision cochlear implant surgery in adults and children. The Laryngoscope 2009; 119: 152-157.

14. Taibah $\mathrm{K}$. The transmeatal approach: a new technique in cochlear and middle ear implants. Cochlear Implants Int. 2009; 10(4): 218-228.

15. Tange RA. Modifications on the Alternative Method for Cochlea Implantation, Cochlear Implant. Research Updates, Dr. Cila Umat (Ed.) 2012; ISBN: 978-953-510582-4.

16. Fayad JN, Georges WB. , Micheletto JN, and Parisier SC. Facial Nerve Paralysis Following Cochlear Implant Surgery. Laryngoscope 2003; 113(8):1344-1346.

17. Ariel Moctezuma, Jane Tu .An Overview of Cochlear Implant Systems. Otoneurology 2011;.27-31. 